\title{
Overexpression of Annexin A2 Receptor Inhibits Neovascularization via the Promotion of Krüppel-Like Transcription Factor 2
}

\author{
Ting Guo Hongyuan Song $^{\mathrm{a}}$ Zichang Zhao ${ }^{\mathrm{a}} \quad$ Zhongtian $\mathrm{Qi}^{\mathrm{b}} \quad$ Shihong Zhao \\ aDepartment of Ophthalmology, Changhai Hospital, Second Military Medical University, Shanghai, \\ ${ }^{b}$ Department of Microbiology, Shanghai Key Laboratory of Medical Biodefense, Second Military \\ Medical University, Shanghai, China
}

\section{Key Words}

Annexin A2 receptor - Human Retinal Endothelial Cells $\cdot$ Human Vein Umbilical Endothelial Cells $•$ Neovascularization $・$ Krüppel-like transcription factor 2

\begin{abstract}
Background/Aims: Annexin A2 receptor (AX2R) can mediate annexin A2 signalling and induce apoptosis in a variety of cells, but its role in neovascularization (NV) remains unclear. Krüppel-like transcription factor 2 (KLF2) is known to be expressed in a range of cell types and to participate in a number of processes during development and disease, such as endothelial homeostasis, vasoregulation and vascular growth/remodelling. The aim of our study was to investigate the role of AX2R in NV and the plausible molecular mechanism. Methods: We constructed a eukaryotic overexpression plasmid for AX2R (Lenti-AX2R) by using polymerase chain reaction (PCR). The full-length human AX2R gene was transfected into human retinal endothelial cells (HRECs) and human umbilical vein endothelial cells (HUVECs) using lentivirus vectors to overexpress AX2R. All experiments were divided into three groups: control, negative control (Lenti-EGFP), and Lenti-AX2R. Cell proliferation, cell migration, tube formation, mouse aortic ring assays and mouse matrigel plug assay were applied to analyse the effect of $A X 2 R$ in NV. Furthermore, we conducted flow cytometry to evaluate whether $A X 2 R$ could influence the cell cycle. A series of cell cycle-related proteins including cyclin A1, cyclin B1, cyclin D1, cyclin E1, CDK1, and p-CDC2 were detected by WB. The mRNA and protein levels of KLF2, vascular endothelial growth factor (VEGF) and vascular endothelial growth factor receptor 2 (VEGFR2) were further quantified by RT-PCR and WB to reveal the possible mechanism. Results: Overexpression of AX2R significantly inhibited cell proliferation, migration and tube formation in both types of endothelial cells (ECs), HRECs and HUVECs. It also suppressed vessel sprouting in the mouse aortic ring assay and NV in mouse matrigel plug assay. Furthermore, infection with Lenti-AX2R lentivirus arrested the cell cycle in S/G2 and influenced the expression of a series of cell cycle-related proteins. We also found that the overexpression of AX2R increased the expression of KLF2, mediating VEGF and VEGFR2.

Shihong Zhao

and Zhongtian Qi

Department of Ophthalmology, Department of Microbiology, Shanghai Key Laboratory of Medical Biodefense, Changhai Hospital, Second Military Medical University, Shanghai (China)

Tel. +86-21-31161991, E-Mail zhaosh@smmu.edu.cn, qizt@smmu.edu.cn
\end{abstract}

KARGER 
Conclusions: Overexpression of AX2R contributes to the inhibition of NV via suppressing KLF2 ubiquitin-dependent protein degradation, which might therefore be a therapeutic option for NV. It could be considered more broadly as an anti-angiogenic agent in the treatment of neovascular-related diseases in the future.

\section{Introduction}

$\mathrm{NV}$, which is the growth of new blood vessels from pre-existing ones, is a highly complex process [1]. In many physiological and pathological processes, such as in wound healing, retinal NV (RNV) and tumour metastasis, NV plays different roles [2, 3]. RNV is one of the main causes of blindness in numerous ocular neovascular diseases including proliferative diabetes mellitus (PDR) and retinopathy of prematurity [4, 5]. The current methods of treatment for RNV are limited. They can improve vision and pathological conditions to some extent; however, they fail to achieve the desired effects [6, 7]. Therefore, it is important and urgent to further explore new targets for the prevention and treatment of adverse effects of NV.

VEGF is well known as the main target for the treatment of ocular neovascular disease. VEGF-related drugs have been used in clinical applications [8]. Over the past several decades, work from several laboratories has shown that annexin A2 plays an important role in NV by interacting with VEGF $[9,10]$. Furthermore, work on annexin A2/AX2R in regulation of $\mathrm{NV}$ has been reported. Specifically, AX2R is a new vascular regulatory molecule that is considered to be a receptor for annexin A2 [11]. AX2R encoding a 193 amino acid protein was obtained from cDNA clones of bone marrow cells. It is located in chromosome 5 , open reading frame $39[12,13]$. AX2R was originally discovered in 2006 and is involved in the formation of bone marrow stromal cells [12]. AX2R exists on the cell surface and can interact with annexin A2 to promote cancer cell adhesion, migration, and proliferation [14, 15]. However, recent studies have found that AX2R is also present in the cytoplasm and the nucleus, and it can induce apoptosis independently of annexin A2 $[15,16]$. Disrupting the expression of AX2R can inhibit proliferation of HUVECs, while overexpression of AX2R is able to induce apoptosis in choroidal melanoma cells $[16,17]$. These findings increase our interest in investigating the role of AX2R in NV.

Ubiquitin-dependent protein degradation is involved in various biological processes by regulating protein abundances [18]. KLF2, which includes 355 amino acids, has an automatic suppression region in its first 110-167 amino acids that is capable of binding to the ubiquitin ligase WW domain. This domain contains E3 ubiquitin protein ligase 1 (WWP1), and binding leads to KLF2 protein ubiquitination and degradation $[19,20]$. Studies indicate that KLF2 can activate endothelial inflammation by upregulating the expression of endothelial nitric oxides (eNOs) through the NF-kB pathway [21, 22]. Meanwhile, KLF2 binds to the VEGFR2 promoter to inhibit migration and expression of ECs [23]. However, the relationship between and function of AX2R and KLF2 in NV remain to be investigated.

\section{Materials and Methods}

Materials and reagents

Foetal bovine serum (FBS), penicillin and streptomycin (P/S), Dulbecco's Modified Eagle's Medium (DMEM), Lipofectamine ${ }^{\mathrm{TM}}$ 2000, TRIzol reagent, rabbit/mouse secondary antibody and Opti-MEM were purchased from Invitrogen (Shanghai, China). Endothelial cell medium (ECM) and endothelial cell growth supplement (ECGS) were purchased from Sciencell (San Diego, USA). Antibodies against AX2R, KLF2, VEGF, and VEGFR2 were purchased from Abcam (Cambridge, United Kingdom). An antibody against GAPDH was purchased from Abclonal (Boston, USA). Antibodies for cell cycle related-protein, including cyclin A1, cyclin B1, cyclin D1, cyclin E1, CDK1, and p-CDC2, were purchased from Proteintech ${ }^{\mathrm{TM}}$ (Wuhan, China). 


\section{Cellular Physiology Cell Physiol Biochem 2018;46:1617-1627

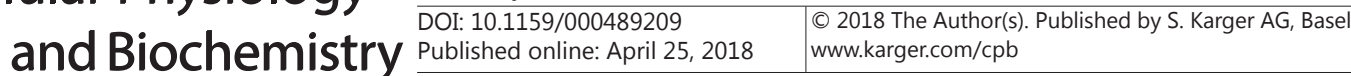 Guo et al.: AX2R Inhibits Neovascularization}

PrimeScript ${ }^{\mathrm{TM}}$ RT Master Mix and SYBR $®$ premix Ex Taq ${ }^{\mathrm{TM}}$ were purchased from TaKaRa (Dalian, Japan). Transwell chambers for the assay migration were obtained from Corning Costar (Herndon, USA) and Matrigel matrix for tube formation was purchased from BD Biosciences (San Jose, USA). A Cell-Light ${ }^{\mathrm{TM}}$ Edu Apollo567 In vitro Kit was obtained from RIB \& BIO (Guangzhou, China). PI staining buffer for cell cycle analysis was obtained from Sungene Biotech (Tianjin, China). Wild-type (WT) C57BL/6 mice were purchased from Animal Experimental Center of Second Military Medical University (Shanghai, China). VEGF 165 was obtained from Peprotech (New Jersey, USA).

\section{Cell culture}

HRECs were purchased from Sciencell (San Diego,

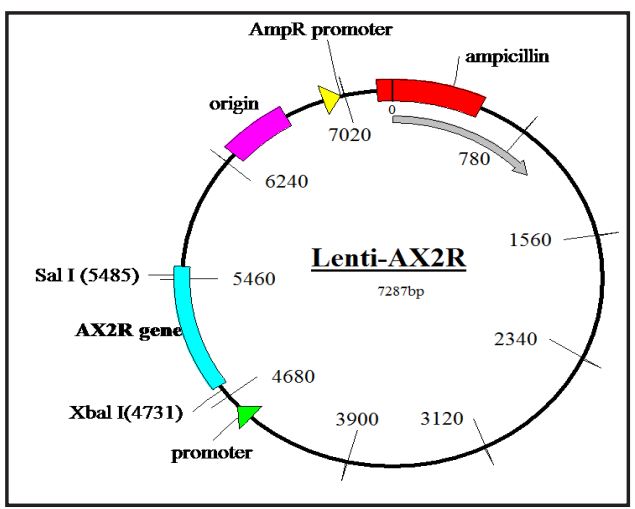

Fig. 1. Map of Lenti-AX2R. The blue curve represents the AX2R cDNA. The AX2R cDNA was inserted between the XbaI and SalI sites. USA), cultured in ECM containing 5\% FBS, 1\% ECGS and 1\% P/S. HUVECs and 293T cells were purchased from American type culture collection (ATCC, Manassas, USA) and were cultured DMEM medium containing 10\% FBS, $1 \% \mathrm{P} / \mathrm{S}, 1 \%$ non-essential amino acids (NEAA) and $1 \%$ glycine. Cells that had been identified by cell short tandem repeat (STR) genotyping were cultured at $37{ }^{\circ} \mathrm{C}$, in a $5 \% \mathrm{CO}_{2}$ cell incubator.

\section{Lenti-AX2R plasmid vector construction}

The vector used was pRRLsin.PPTs.hCMV.EGFPpre. TRIzol reagent was used to extract total RNA from Human retinal endothelial cells (HRECs). Then, the RNA was reverse-transcribed to cDNA with a reverse transcription kit for reverse-transcription polymerase chain reaction (RT-PCR). The cDNA was used for polymerase chain reaction (PCR). The human AX2R primer sequences for the PCR were as follows: forward, 5' -TCT AGA GCC GCC ACC ATG GAG CAA CAT TTT CTT GGC-3' and reverse, 5'-GTC GAC CTA AGG CTG CTT AGC TCC ACA GAT CCG-3'. The AX2R PCR product that was ligated into the pEasy -Blunt Cloning Vector (pEasy Blunt Cloning Kit; TRAN, Shanghai, China) was 582 bp in length and was inserted between the XbaI and SalI sites in the vector. The Lenti-AX2R plasmid (Fig. 1) was extracted with a QIAprep spin Miniprep Kit (QIAGEN, Dusseldorf, Germany). Sequencing and comparison with the GenBank sequence (NM 001014279.2) were performed to confirm the identity of Lenti-AX2R.

\section{Infection of HRECs and HUVECs with lentivirus}

Lenti-AX2R, a recombinant lentivirus vector encoding human AX2R, was generated by cloning cDNA encoding the total length of human AX2R into the packaging plasmid of MDL, VSVG, REV. Cells cultured to approximately $20 \%$ confluence were infected with the recombinant lentivirus vector (50 multiplicity of infection) for approximately 48-72 hours to ensure infection efficiency of up to 70-80\% before further treatment. The parental lentivirus vector Lenti-EGFP was used as a negative control.

\section{Cell proliferation assay}

HRECs and HUVECs were grown in a 48-well plate overnight, and then, Lenti-AX2R lentivirus was added to the wells. Lenti-EGFP lentivirus was added to additional wells as a negative control. The cells were stained after 72 hours using a Cell-Light ${ }^{\mathrm{TM}}$ Edu Apollo567 In vitro Kit according to the manufacturer's protocol and observed using a fluorescence microscope (Olympus, Osaka, Japan).

\section{Cell migration assay}

The migration assay was performed with a transwell chamber. In brief, $3 \times 10{ }^{5}$ cells infected with lentivirus were plated in $100 \mu \mathrm{L}$ medium in each chamber. After incubation for 12-16 hours, cells that had migrated to the lower surface of the membrane were fixed with $4 \%$ paraformaldehyde and stained with crystal violet dye for 2 hours. The stained cells were imaged and counted in 10 high-power fields (Olympus, Osaka, Japan). 


\section{Cellular Physiology Cell Physiol Biochem 2018;46:1617-1627 \\ and Biochemistry \begin{tabular}{c|c} 
DOI: 10.1159/000489209 & $\begin{array}{l}\text { O 2018 The Author(s). Published by S. Karger AG, Basel } \\
\text { www.karger.com/cpb }\end{array}$ \\
\cline { 2 - 3 }
\end{tabular}}

Guo et al.: AX2R Inhibits Neovascularization

\section{Tube formation assay}

Matrigel $(150 \mu \mathrm{L})$ was added to each well of a 48-well plate. HRECs in ECM medium and HUVECs in DMEM were plated at $3 \times 10^{5}$ cells/well and cultured for 4 hours. Tube formation was examined using an inverted microscope equipped with a digital camera (Leica, Wetzlar, Germany). Tube-like structures exceeding 3 joint points were counted.

\section{Mouse aortic ring assay}

The aortas of adult WT C57BL/6 mice (8-12 weeks of age) were dissected and cut into aortic rings . Aortic segments that were placed in a matrigel-covered 96-well plate for 6 days were infected with lentiviral vectors. A crude view of microvascular sprouts was sufficient to identify and count vessels sprouting during the experiment using a phase-contrast microscope (Leica, Wetzlar, Germany).

\section{Matrigel plug assay}

Lentiviral Lenti-EGFP or Lenti-AX2R was mixed in the matrigel solution at $4{ }^{\circ} \mathrm{C}$ with $100 \mathrm{ng} / \mathrm{ml} \mathrm{VEGF165}$ and 20 units of heparin. Then, $500 \mu$ of matrigel containing lentiviruses was injected subcutaneously into the abdomen of 6 -week-old C57BL/6 male mice. After 6 days, the skin of each mouse was pulled off to expose an intact matrigel plug. Thereafter, haematoxylin and eosin (HE) staining was used to identify the formation and infiltration of new functional microvessels. Functional microvessels with intact RBCs were quantified manually using a microscope.

\section{Cell Cycle assay}

Propidium iodide (PI) analysis was used to detect DNA markers. Cells infected with lentivirus were fixed in $70 \%$ ice anhydrous ethanol for 2 hours at $4{ }^{\circ} \mathrm{C}$ and then stained for 30 minutes using PI at room temperature. The samples were analysed using a MACSQuant Analyzer (MiltenyiBiotec, Teterow, Germany). The data were then analysed using Modfit software.

$W B$

Cells were lysed with RIPA buffer on ice for 15 minutes, boiled for $15 \mathrm{~min}$, centrifuged at 12, $500 \mathrm{rpm}$ for 5 minutes, and then stored at $-20^{\circ} \mathrm{C}$.. The protein content was quantified (EZQ ${ }^{\circledR}$ assay, Life Technologies), and $50 \mu \mathrm{g}$ of each sample was resolved by SDS-PAGE on a $12.5 \%$ Mini-Protean ${ }^{\circledR}$ TGX Stain-Free ${ }^{\mathrm{TM}}$ Gel (BioRad, Hercules, USA) and transferred to PVDF membranes using the Trans-Blot ${ }^{\circledR}$ Turbo ${ }^{\mathrm{TM}}$ Transfer Pack and System (Bio-Rad, Hercules, USA). The membranes were blocked with TBST containing 5\% skim milk for 2.5 hours and incubated with primary antibody overnight at $4^{\circ} \mathrm{C}$.. Following three washes in TBST, the membranes were incubated with anti-rabbit/mouse/goat IgG HRP secondary antibody $(1 / 2500)$ at room temperature for 2.5 hours. Chemiluminescence substrate was applied (Clarity ${ }^{\mathrm{TM}}$ Western ECL Blotting Substrate, Bio-Rad, Hercules, USA), and the blots were analysed using the ChemiDoc ${ }^{\mathrm{TM}}$ Touch Imaging System (Bio-Rad, Hercules, USA).

\section{RT-PCR}

Cells at $80-90 \%$ confluence were harvested, and RNA was extracted using TRIzol reagent on ice. The total RNA concentration was quantified using a NanoDrop 2000 spectrophotometer (Thermo Scientific, Waltham, MA, USA). The RNA (500 ng) was reverse transcribed using PrimeScript ${ }^{\mathrm{TM}}$ RT Master Mix. Quantification PCR was performed using SYBR ${ }^{\circledR}$ premix Ex $\mathrm{Taq}^{\mathrm{TM}}$. The primer sequences are shown in Table 1 . The results were calculated according to the $2^{-\Delta \Delta c t}$ relative quantification method.

\section{Data analysis}

All experiments were repeated three times independently, and the data were analysed using GraphPad Prism 5 software and are presented as the
Table 1. Primer Sequences

\begin{tabular}{lc}
\hline Name & primer sequences $\left(5^{\prime}{ }^{\prime} 3^{\prime}\right)$ \\
\hline GAPDH & Forward ACCACAGTCCATGCCATCAC \\
& Reverse TCCACCACCCTGTTGCTGTA \\
AX2R & Forward CGGAGTCTACTGGCAAAACG \\
& Reverse GCCTTCTGCTGCTATCTAAG \\
VEGF & Forward CCGTCCTGTGTGCCCCTAATG \\
& Reverse CGCATGATCTGCATAGTGACGTTG \\
VEGFR2 & Forward CCAGCAAAAGCAGGGAGTCTGT \\
& Reverse TGTCTGTGTCATCGGAGTGATATCC \\
KLF2 & Forward TGCGGCAAGACCTACACCAAGAGT \\
& Reverse AGCCGCAGCCGTCCCAGTT
\end{tabular}




\section{Cellular Physiology Cell Physiol Biochem 2018;46:1617-1627

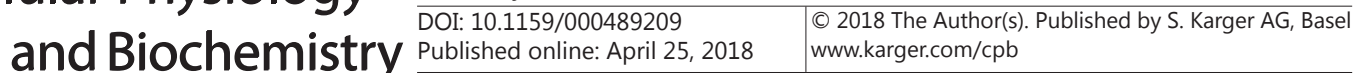 \\ Guo et al.: AX2R Inhibits Neovascularization}

means and standard deviation (mean+SD). Statistical analysis was performed with a one-way ANOVA Tukey test. A significance level of $P<0.05$ was considered to be statistically significant.

\section{Results}

Overexpression of AX2R suppresses HREC and HUVEC proliferation

We constructed a eukaryotic overexpression plasmid for AX2R (Lenti-AX2R) to determine whether AX2R plays a role in NV. We first compared the proliferation abilities among the Lenti-AX2R, Lenti-EGFP and control groups. The experimental results demonstrated that in the HREC (Fig. 2A) and HUVEC (Fig. 2C) populations, cells infected with Lenti-AX2R lentivirus for 48-72 hours had the slowest growth . Furthermore, the number of duplicate cells stained with $1 \times$ Apollo was also dramatically decreased for both HRECs and HUVECs (Figs. 2A, 2C). There were significant differences between the Lenti-EGFP and Lenti-AX2R groups. Moreover, the statistics indicated a remarkable decrease in cell proliferation in the experimental group (Fig. 2B, Fig. 2D).

\section{Overexpression of AX2R suppresses HREC and HUVEC migration}

Furthermore, we investigated whether AX2R affects endothelial cell migration, which is a vital process for NV. After incubation for 12-16 hours in a transwell chamber, fewer cells were observed on the other side of the chamber in the Lenti-AX2R group (HRECs, Fig. 3A; HUVECs, Fig. 3C). In addition, the state of cells infected with Lenti-AX2R lentivirus worsened, and their shape was not round any longer. The statistical analysis indicated that the number of migrated cell in the AX2R overexpression group were lower than that in the other groups for both HRECs (Fig. 3B) and HUVECs (Fig. 3D).

\section{Overexpression of AX2R suppresses HREC and HUVEC tube formation}

The role of AX2R in NV was further evaluated using a tube formation assay. We detected that HRECs (Fig. 4A) and HUVECs (Fig. 4C) infected with AX2R lentivirus had difficulty forming a complete tubular structure compared with other groups. As shown in Fig. 4A

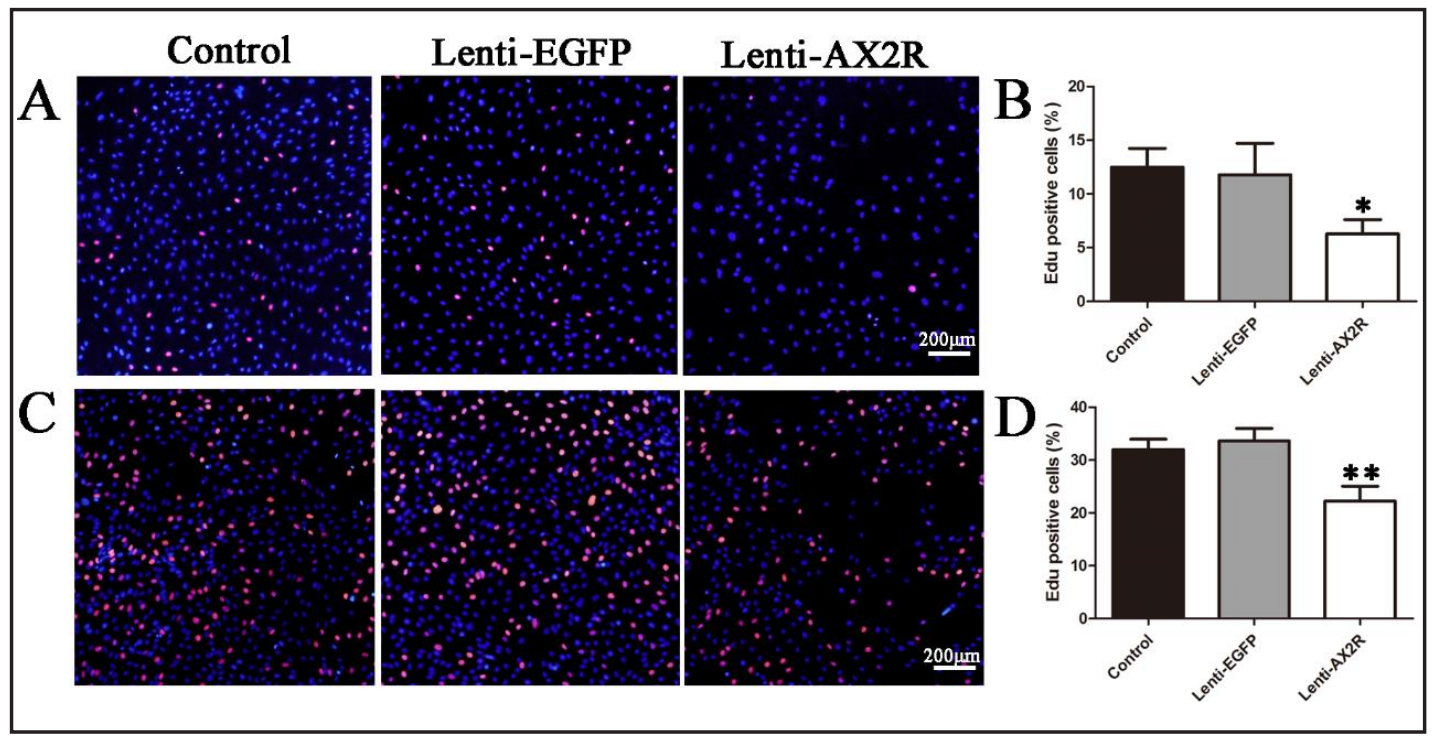

Fig. 2. Representative image of replicating HRECs and HUVECs (magnification, $\times 100$ ). HRECs and HUVECs stained with $1 \times$ Hoechst33342 as a nuclear marker (blue), and the replicating cells stained with $1 \times$ Apollo (red). (A, C) Cells in the proliferative phase accounted for all cells in the random field of vision for HRECs (A) and HUVECs (C). (B, D) Quantification of Edu-positive cells in A and C, respectively. The data are presented as the mean and standard deviation $(\mathrm{n}=3),{ }^{*} \mathrm{P}<0.05,{ }^{* *} \mathrm{P}<0.01$. 
Fig. 3. Lenti-AX2R inhibited the migration of ECs. (A, C) Representative pictures of the migrated HRECs (A, magnification, $\times 100$ ) and HUVECs (C, magnification, $\times 200$ ). (B, D) $B$ and $D$ are quantified, and the data are presented as a histogram $(\mathrm{n}=3),{ }^{* * *} \mathrm{P}<0.001$.

Fig. 4. Overexpression of $\mathrm{AX} 2 \mathrm{R}$ inhibited tube formation by ECs. (A, C) HRECs (A) and HUVECs (C) infected with Lenti-AX2R were placed in plates coated with matrigel, and tubular structures were imaged $(n=3)$. (B, D) Quantification data of $\mathrm{A}$ and $\mathrm{C} .{ }^{* *} \mathrm{P}<0.01$; $* * * \mathrm{P}<0.001$.

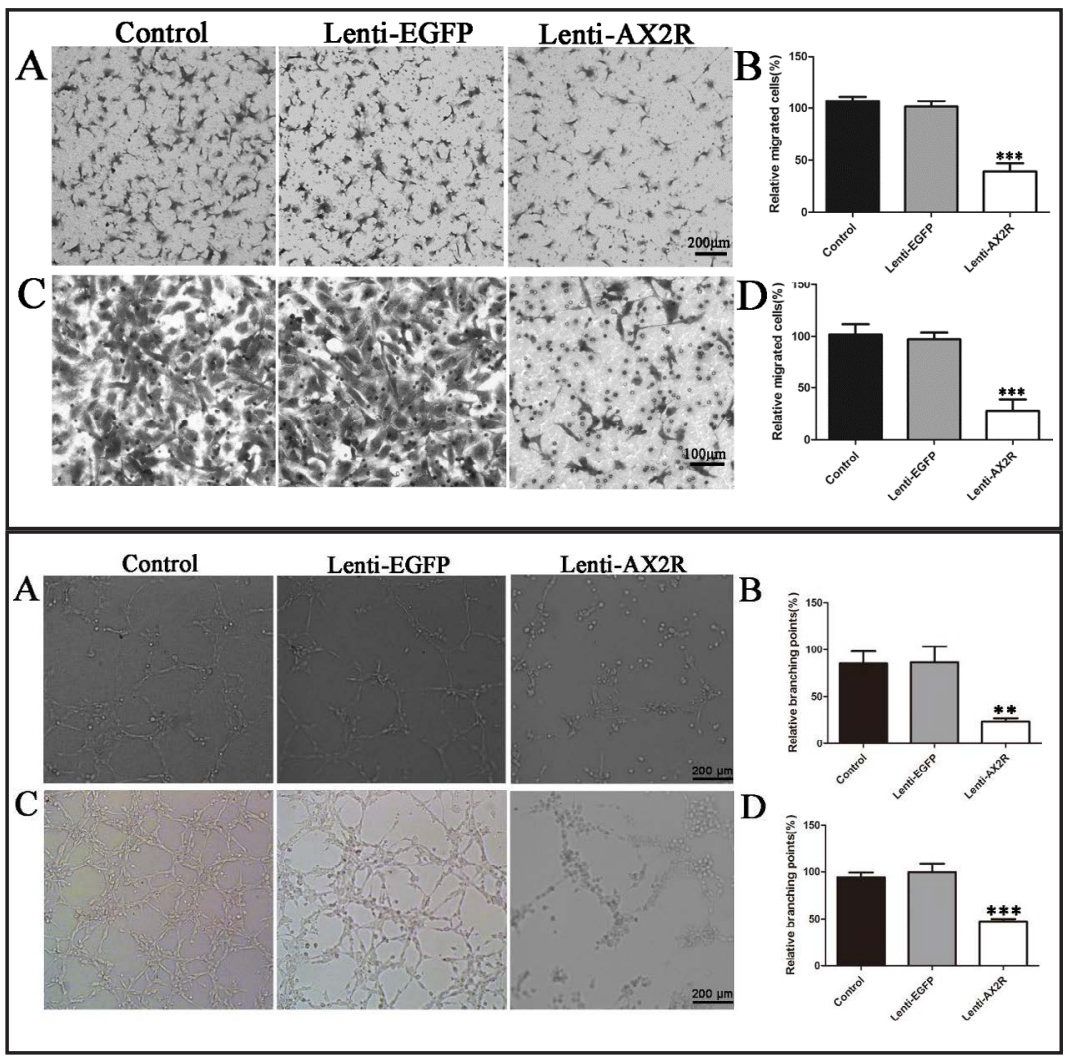

and Fig. 4C, the cells themselves infected with Lenti-AX2R lentivirus were contracted and rounded, which might be a reason that they could not form a complete tubular structure. Statistical results showed a remarkable decrease in the tube formation ability in the experimental group (HRECs, Fig. 4B; HUVECs, Fig. 4D).

\section{Overexpression of AX2R inhibits $N V$ in mouse aortic ring assay}

To better investigate the role of AX2R in NV, we tested the effect of AX2R overexpression on vessel sprouting using a mouse aortic ring assay. After 7-10 days, optimal vessel sprouting could be observed in the mouse aortic ring assay treated with endothelial growth factors, which grew in a line or interweaving a network around the mouse aortic ring on the matrigel. Vessel sprouting could be observed clearly in the control and Lenti-EGFP groups (Figs. 5A, $5 B)$. However, it was difficult to observe complete vessel sprouting around the mouse aortic ring in the Lenti-AX2R group (Fig. 5C). Moreover, the data showed that AX2R overexpression could suppress mouse aortic ring sprouting significantly at day 8 compared with the other groups (Fig. 5D). The numbers and lengths of sprouting vessels were further analysed using ImageJ software and presented in a histogram (Fig. 5D).

\section{Overexpression of AX2R inhibits $N V$ in mouse matrigel plug assay}

To determine whether AX2R is important to $N V$, we studied the effect of AX2R overexpression on the neovascular response using a matrigel plug assay in vivo. We found that matrigel plugs containing Lenti-EGFP virus appeared red and were filled with RBCs 6 days after implantation, indicating that functional vascular structures were induced within the matrigel (Fig. 6Aa, 6Ab, 6Ac). In contrast, the addition of Lenti-AX2R virus notably inhibited VEGF165-induced vascular growth, and the matrigel plugs were pale due to the lack of RBCs (Fig. 6Aa, 6Ad). The NV was confirmed by immunohistochemistry using antiCD31 antibody, which is a specific marker for ECs. A reduction of NV was observed in the presence of Lenti-AX2Rvirus (Fig. 6B). Collectively, these data suggest that AX2R is a key regulator of $\mathrm{NV}$ in vivo.

\section{KARGER}




\section{Cellular Physiology Cell Physiol Biochem 2018;46:1617-1627 \begin{tabular}{l|l} 
DOI: 10.1159/000489209 & $\begin{array}{l}\text { O 2018 The Author(s). Published by S. Karger AG, Basel } \\
\text { www.karger.com/cpb }\end{array}$
\end{tabular} \\ Guo et al.: AX2R Inhibits Neovascularization}

AX2R influences HREC and HUVEC cell cycle distribution

The cell cycle is a complicated process that determines cell division and duplication. Since AX2R overexpression could inhibit proliferation of HRECs and HUVECs, we examined whether it affected the cell cycle using flow cytometry. The result showed that the number of cells in G1 phase was invariable. However, the amount of cells in $\mathrm{S}$ phase increased, and the amount in G2 phase decreased significantly in the Lenti-AX2R group compared to that in the other groups (HRECs, Figs. 7A, 7B; HUVECs; Figs. 7C, 7D). The results suggested that AX2R overexpression could prevent cells from transiting into $\mathrm{G} 2$ phase from $\mathrm{S}$ phase. Meanwhile, a series of cell cycle-related proteins including cyclin A1, cyclin B1, cyclin D1, cyclin E1, CDK1, and p-CDC2 were examined. Among them, the expression of cyclin B1 and cyclin E1 was almost unchanged, but AX2R overexpression increased the expression of p-CDC2 and inhibited the expression of cyclin A1 and cyclin E1 (HRECs, Fig. 7E; HUVECs, Fig. 7F).

\section{AX2R influenced the functions of HRECs and HUVECs by promoting KLF2}

Previous studies indicated that overexpression of AX2R could induce apoptosis in multiple cells and induce tumour cell autophagy $[16,17]$. The processes play important roles in ageing, regeneration and the dynamic balance of the body. Meanwhile, the ubiquitindependent protein degradation pathway is involved in both apoptosis and autophagy processes. Our results showed that expression of KLF2 increased at the mRNA and protein levels after ECs were infected with AX2R lentivirus. At the same time, the expression levels of VEGF and VEGFR2 was detected and were decreased significantly in HRECs (Figs. 8A,8B) and HUVECs (Figs. 8C, 8D).

\section{Discussion}

In the present study, we showed that AX2R overexpression could significantly suppress the proliferation, migration and tube formation of ECs (Figs. 2, 3, 4,). Furthermore, AX2R overexpression could suppress vessel sprouting in a mouse aortic ring assay and NV in mouse matrigel plugs (Fig. 5, 6). These data indicated that AX2R might inhibit NV. The cell cycle contains four major phases: G1, S, G2 and M. Cells must experience the whole cycle to proliferate. Since AX2R overexpression could inhibit cell proliferation (Fig. 2), we further examined whether it is relevant to the cell cycle. We found that compared to other groups, HRECs and HUVECs in the AX2R overexpression group were obviously arrested in S/G2 phase (Figs. 7A-D). The expression of cyclin A1, cyclin B1, cyclin D1, cyclin E1, CDK1 and p-CDC2 further proved that AX2R overexpression could interfere in the cell cycle process (Figs. 7E, 7F). Experimental results identified that AX2R, a newly discovered gene, is possibly a functional target of NV.

We provided evidence showing that AX2R overexpression could suppress NV. Although many studies have shown that AX2R is a specific surface receptor for AXNA2, the result we obtained was different [11]. First, the role of AX2R is well known as a receptor for annexin 
Fig. 6. The overexpression of AX2R inhibited VEGF165-induced neovascularization in vivo. (A-a) The neovascularization in the intact matrigel plugs was examined after 6 days $(n=5)$. Plug sections of $5 \mu \mathrm{M}$ were immunostained with an antiCD31 antibody specific for blood vessels (A-b, $c, d)$. The images were captured using a Leica DM 4000B microscope (magnification, 200×).

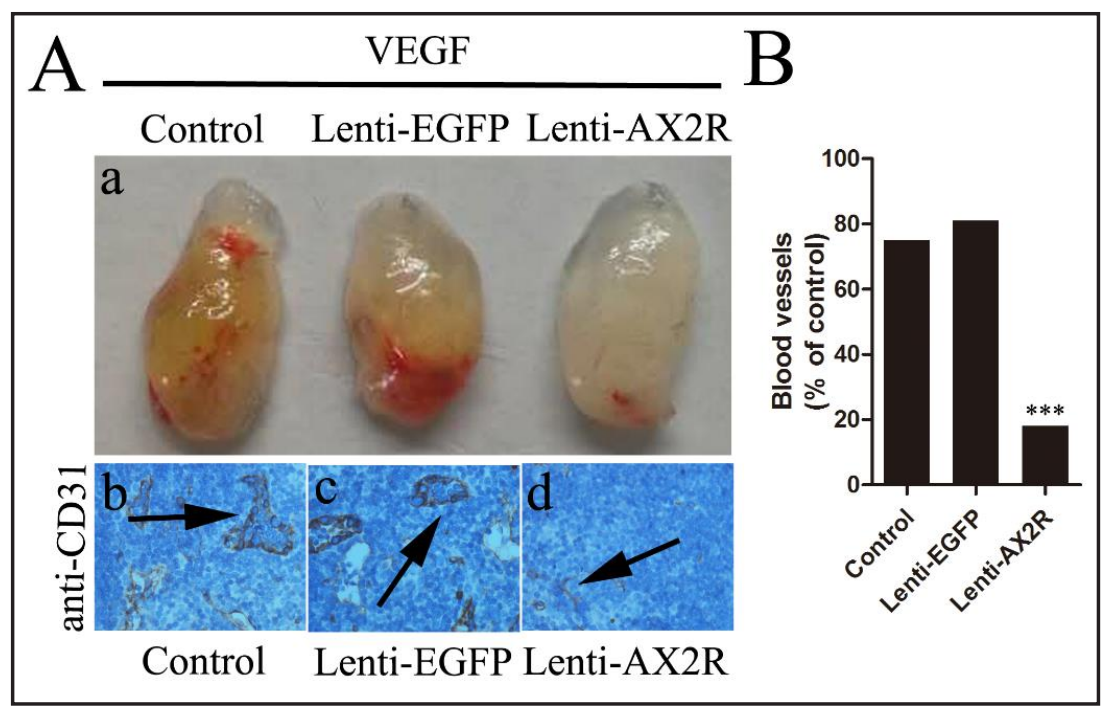
Black arrows, blood vessels. (B)The statistics indicated a reduction of neovascularization in the presence of Lenti-AX2R virus.

Fig. 7. Lenti-AX2R influenced HREC and HUVEC cell cycle distribution. (A, C) Representative images of the cell cycle in HRECs (A) and HUVECs (C). (B, D) The data (mean+SD) for G1, S and G2 were quantified and are presented as a histogram $\quad(n=3)$. ${ }^{*} \mathrm{P}<0.05 ; \quad{ }^{* *} \mathrm{P}<0.01$. (E, F) Detection of cell cycle-related proteins in HRECs (E) and HUVECs (F) using WB.

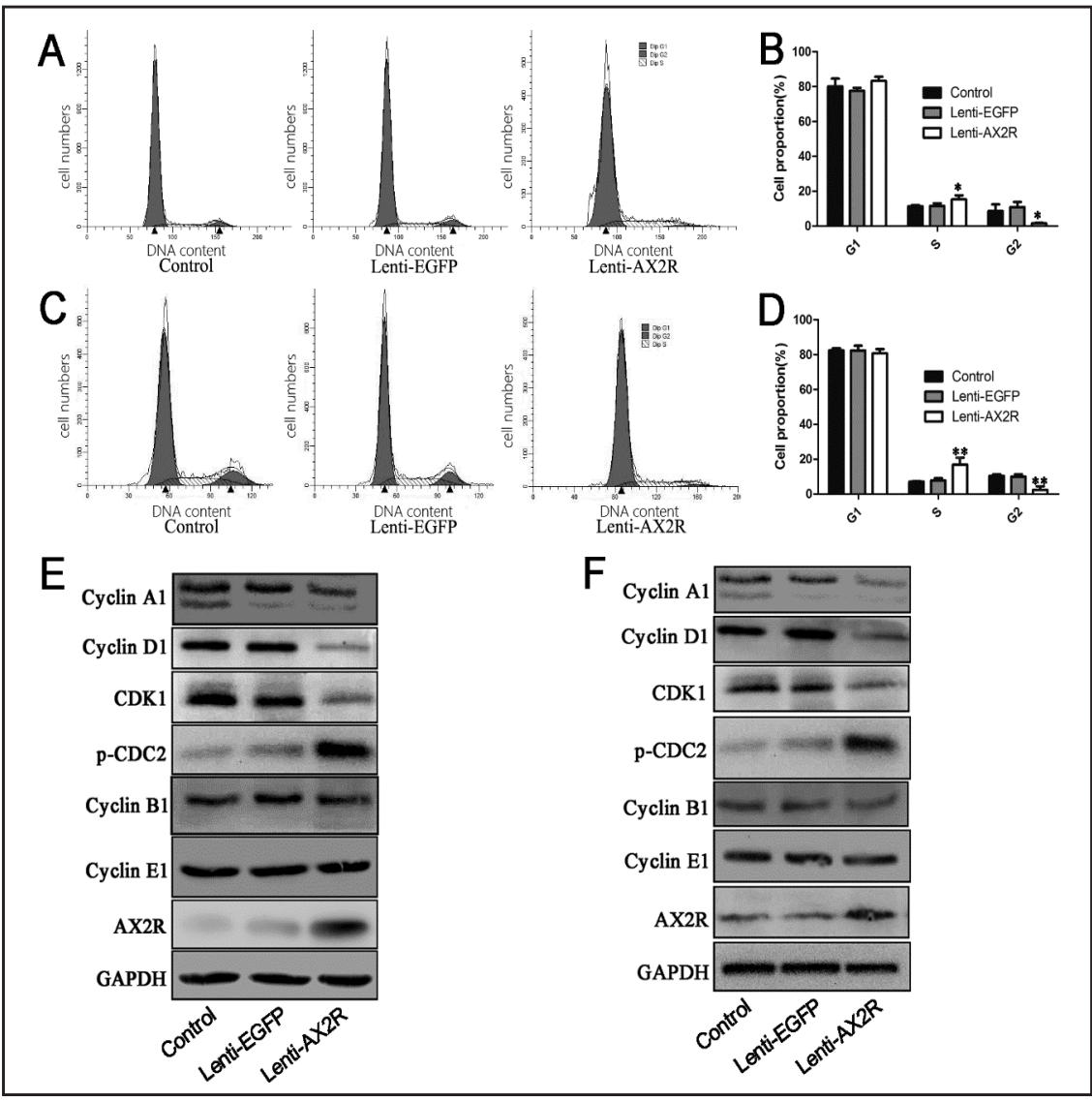

A2 in NV, particularly in tumour diseases [24]. Overexpression of annexin A2, binding and co-localization with S100A10 mediated S100A10 to promote tumour invasion, metastasis, and angiogenesis [25]. However, our results indicated that overexpression of AX2R inhibited the process of $\mathrm{NV}$, suggesting $\mathrm{AX} 2 \mathrm{R}$ might not enhance the role of annexin A2 in NV at all. This finding is consistent with previous reports that AX2R could also be located in the 


\section{Cellular Physiology Cell Physiol Biochem 2018;46:1617-1627

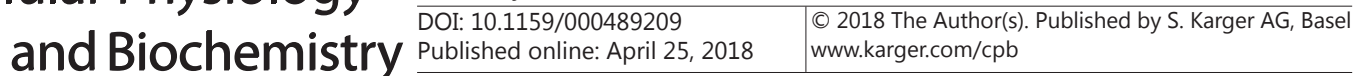 \\ Guo et al.: AX2R Inhibits Neovascularization}

Fig. 8. Detection of KLF2 molecular signalling pathway. $(\mathrm{A}, \mathrm{C})$ The mRNA expression levels of KLF2, VEGFR2 and VEGF were detected using RT-PCR in HRECs (A) and HUVECs (C). (B, D) The protein levels of VEGF, VEGFR2 and KLF2 were detected by WB. The data are statistically significant $(n=3)$. $* * \mathrm{P}<0.01$.

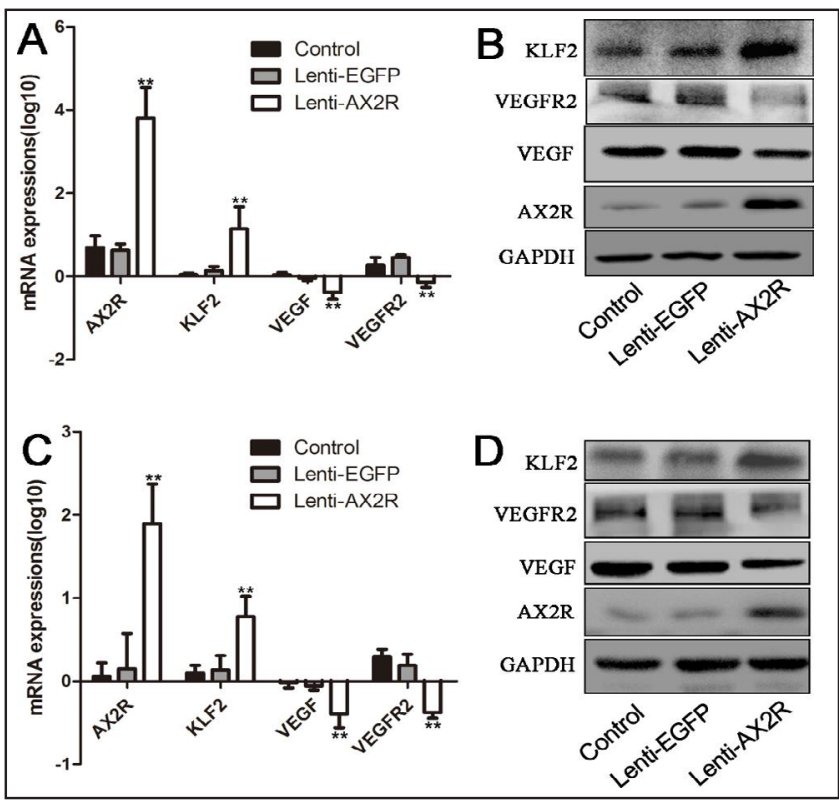

cytoplasm and act as a novel inducer of apoptosis independently of AXNA2 in human cells, partially through activating Caspase-8 in a manner that is different from the conventional apoptotic pathway [15]. AX2R was able to induce apoptosis and autophagy in Mum2C cells [17], which further confirms our conclusion. Furthermore, it is worthy of consideration that the transcriptional level of AX2R in multiple cell types was considerably high, while the translational level of AX2R could hardly be detected [15]. This topic is still worthy of discussion. The previous study showed that disrupting AX2R was able to inhibit NV via inactivating phosphorylation of the AKT and ERK pathways [16]. However, our results showed that overexpression of AX2R could also suppress NV. This is not a contradictory result. This study showed that the expression of AX2R might maintain a balanced status in ECs. Our results also clearly demonstrated that AX2R can interfere in the cell cycle process to influence NV; however, the detailed potential mechanism remains worth exploring.

To better understand the process of Lenti-AX2R mediated suppression of NV, we investigated KLF2-related ubiquitin-dependent protein degradation, which was unintentionally discovered when Lenti-EGFP-AX2R (a fusion plasmid) was observed in HRECS and 293T cells. The protein of Lenti-EGFP-AX2R accumulated intracellularly in HRECs and 293T cells, which was possibly related to the protein degradation signal pathway. KLF2 is a central regulator of endothelial functions that plays a critical role in angiogenesis, vascular tone, inflammation and thrombosis [26, 27], and it is regulated through the ubiquitin-dependent protein degradation pathway at the posttranslational level in endothelial cells [28]. A previous study showed that the E3 ubiquitin ligase WWP1 inhibits KLF2 transcriptional activity and targets KLF2 for ubiquitination and degradation in a ubiquitin ligase activity independent manner [23]. The E3 ubiquitin ligase Smurf1can target KLF2 for degradation in H1299 cells [29]. Our data showed that KLF2 protein expression levels increased when HRECs and HUVECs were infected with AX2R lentivirus. Evidence shows that KLF2 destruction depends on the phosphorylation of KLF2 via glycogen synthase kinase-3 (GSK3) at two conserved phosphodegrons. Overexpression of AX2R might mutate these phosphodegron motifs, abolishing the degradation and ubiquitination of KLF2 $[23,30]$. Although studies have shown that KLF2 mRNA expression is regulated by various signals, including stress, growth factors and cytokines in endothelial cells, the mechanism that regulates KLF2 abundance at the posttranslational level has remained largely unknown $[31,32]$. How KLF2 protein stability is regulated in endothelial cells has also remained unknown. All the above provide a novel mechanism for the function of KLF2 in ECs. Previous investigations indicated that KLF2 is involved in the process of NV via regulating 


\section{Cellular Physiology Cell Physiol Biochem 2018;46:1617-1627

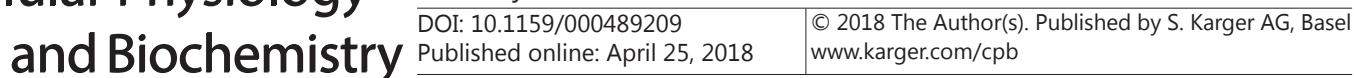 \\ Guo et al.: AX2R Inhibits Neovascularization}

the expression of VEGF and VEGFR2 [23]. This finding is consistent with what we have observed. These data further proved that overexpression of AX2R could activate a KLF2related ubiquitin-dependent protein degradation pathway to contribute to NV inhibition.

\section{Conclusion}

AX2R overexpression could apparently inhibit proliferation, migration, tube formation, vessel sprouting in mouse aortic rings and $\mathrm{NV}$ in mouse matrigel plugs. Furthermore, the possible mechanism might include the inhibition a KLF2 ubiquitin-dependent protein degradation pathway. At the same time, our results indicated that AX2R overexpression could arrest the cell cycle in S/G2. All evidence showed that AX2R overexpression might be a potential option to treat neovascularization-associated diseases in the near future.

\section{Disclosure Statement}

No conflict of interests exists.

\section{Acknowledgements}

This work was supported by the National Natural Science Foundation of China (No. 81470652).

\section{References}

-1 Jetten N, Verbruggen S, Gijbels, MJ, Post MJ, De Winther MP, Donners MM: Anti-inflammatory M2, but not pro-inflammatory M1 macrophages promote angiogenesis in vivo. Angiogenesis 2014;17:109-118.

-2 Do DV, Wang X, Vedula SS, Marrone M, Sleilati G, Hawkins BS, Frank RN: Blood pressure control for diabetic retinopathy. Sao Paulo Med J 2015;133:278-279.

-3 Croci DO, Cerliani JP, Dalotto-Moreno T, Mendez-Huergo SP, Mascanfroni ID, Dergan-Dylon S, Toscano MA, Caramelo JJ, Garcia-Vallejo JJ, Ouyang J, Mesri EA, Jun ttila, Bais C, Shipp MA, Slatino M, Rabinovich GA: Glycosylation- dependent lectin-receptor interactions preserve angiogenesis in anti-VEGF refractory tumors. Cell 2014;156:744-758.

4 Sapieha P, Hamel D, Shao Z, Rivera JC, Zaniolo K, Joyal JS, Chemtob S: Proliferative retinopathies: angiogenesis that blinds. Int J Biochem Cell Biol 2010;42:5-12.

5 Kong L, Bhatt AR, Demny AB, Coats DK, Li A, Rahman EZ, Smith OE, Steinkuller PG: Pharmacokinetics of Bevacizumab and Its Effects on Serum VEGF and IGF-1 in Infants With Retinopathy of PrematurityBevacizumab Pharmacokinetics in Infants With ROP. Invest Ophthalmol Vis Sci 2015;56:956961.

6 Martin DF, Maguire MG, Fine SL, Ying GS, Jaffe GJ, Grunwald JE, Toth C, Redford M, Ferris FL: Ranibizumab and bevacizumab for treatment of neovascular age-related macular degeneration: two-year results. Ophthalmology 2012;119:1388-1398.

7 Solomon SD, Lindsley KB, Krzystolik MG, Vedula SS, Hawkins BS: Intravitreal bevacizumab versus ranibizumab for treatment of neovascular age-related macular degeneration: findings from a Cochoursane Systematic Review. Ophthalmology 2016;123:70-77.

8 Matsumoto K, Ema M: Roles of VEGF-A signalling in development, regeneration, and tumours. J Biochem 2014;156:1-10.

-9 Qnishi M, Ichikawa T, KurozumiK, Inoue S, Marue S, Maruo T, Otani Y, Yoshida K, Yoshida K, Michiue H, Antonio Chiocca E, Date I: Annexin A2 regulates angiogenesis and invasion phenotypes of malignant glioma. Brain Tumor Pathol 2015;32:184-194.

10 Genetos DC, Wong A, Watari S, Yellowley CE: Hypoxia increases Annexin A2 expression in osteoblastic cells via VEGF and ERK. Bone 2010;47:1013-1019.

11 Shiozawa Y, Havens AM, Jung Y, Ziegler AM, Pedersen EA, Wang J, Wang J, Lu G, Roodman GD, Loberg RD, Pienta KJ, Taichman RS: Annexin II/annexin II receptor axis regulates adhesion, migration, homing, and growth of prostate cancer. J Cell Biochem 2008;105:370-380. 


\section{Cellular Physiology Cell Physiol Biochem 2018;46:1617-1627 and Biochemistry Published 1159/000489209 $2018 \quad \begin{aligned} & \text { O 2018 The Author(s). Published by S. Karger AG, Basel } \\ & \text { www.karger.com/cpb }\end{aligned}$}

Guo et al.: AX2R Inhibits Neovascularization

12 Lu G, Maeda H, Reddy SV, Kurihara N, Leach R, Anderson JL, Roodman GD: Cloning and characterization of the annexin II receptor on human marrow stromal cells. J Biol Chem 2006;281:30542-30550.

13 Kujala E, Mäkitie T, Kivelä T: Very long-term prognosis of patients with malignant uveal melanoma. Invest Ophthalmol Vis Sci 2003;44:4651-4659.

14 Mussunoor S, Murray G I: The role of annexins in tumour development and progression. J Pathol 2008;216:131-140.

15 Xiong Y, Fan C, Kong L, Dong L, Zhu N, Zhang J, Wang L, Qin T, Shen Y, Chen M: Annexin II receptor induces apoptosis independent of Annexin II. Apoptosis 2013;18:925-939.

16 Song H, Pan D, Sun W, Gu C, Zhang Y, Zhao P, Qi Z, Zhao S: SiRNA directed against annexin II receptor inhibits angiogenesis via suppressing MMP2 and MMP9 expression. Cell Physiol Biochem 2015;35:875884.

17 Zhang Y, Song H, Guo T, Zhu Y, Tang H, Qi Z, Zhao P, Zhao S: Overexpression of Annexin II ReceptorInduced Autophagy Protects Against Apoptosis in Uveal Melanoma Cells. Cancer Biother Radiopharm 2016;31:145-151.

18 Zattas D, Hochstrasser M: Ubiquitin-dependent protein degradation at the yeast endoplasmic reticulum and nuclear envelope. Crit Rev Biochem Mol Biol 2015;50:1-17.

19 Courivaud T, Ferrand N, Elkhattouti A, Kumar S, Levy L, Ferrigno O, Atfi A, Prunier C: Functional characterization of a WWP1/Tiul1 tumor-derived mutant reveals a paradigm of its constitutive activation in human cancer. J Biol Chem 2015;290:21007-21018.

20 Zeng H, Chi H: mTOR and lymphocyte metabolism. Curr Opin Immunol 2013;25:347-355.

-21 Peralta C, Jiménez-Castro MB, Gracia-Sancho J: Hepatic ischemia and reperfusion injury: effects on the liver sinusoidal milieu. J Hepatol 2013;59:1094-1106.

-22 Zohre S, Kazem NK, Abolfazl Mohammad RY, Aliakbar M, Effat A, Zahra D, Hassan D, Nosratollah Z: Trichostatin A-induced Apoptosis is mediated by Krüppel-like factor 4 in ovarian and lung cancer. Asian Pac J Cancer Prev 2014;15:6581-6586.

23 Wang R, Wang Y, Liu N, Ren C, Jiang C, Zhang K, Yu S, Chen Y, Tang H, Deng Q, Fu C, Wang Y, Li R, Liu M, Pan W, Wang P: FBW7 regulates endothelial functions by targeting KLF2 for ubiquitination and degradation. Cell Res 2013;23:803-819.

24 Zhang W, Zhao P, Xu XL, Cai L, Song ZS, Cao DY, Tao KS, Zhou WP, Chen ZN, Dou KF: Annexin A2 promotes the migration and invasion of human hepatocellular carcinoma cells in vitro by regulating the shedding of CD147-harboring microvesicles from tumor cells. PLoS One DOI: 10.1371/journal.

25 Vandevyver S, Dejager L, Tuckermann J, Libert C: New insights into the anti-inflammatory mechanisms of glucocorticoids: an emerging role for glucocorticoid-receptor-mediated transactivation. Endocrinology 2013;154:993-1007.

26 Tetreault MP, Yang Y, Katz JP: Kruppel-like factors in cancer. Nat Rev Cancer 2013;13:701-713.

27 Nayak L, Lin Z, Jain MK: “Go with the flow”: how Krüppel-like factor 2 regulates the vasoprotective effects of shear stress. Antioxid Redox Signal 2011;15:1449-1461.

-28 Guixé-Muntet S, de Mesquita FC, Vila S, Hernández-Gea V, Peralta C, García-Pagán JC, Bosch J, Gracia-Sancho J: Cross-talk between autophagy and KLF2 determines endothelial cell phenotype and microvascular function in acute liver injury. J Hepatol 2017;66:86-94

29 Du JX, Hagos EG, Nandan MO, Bialkowska AB, Yu B, Yang VW: The E3 ubiquitin ligase SMAD ubiquitination regulatory factor 2 negatively regulates Krüppel-like factor 5 protein. J Biol Chem 2011;286:40354-40364.

-30 Bialkowska AB, Liu Y, Nandan MO, Yang VW: A colon cancer-derived mutant of Krüppel-like factor 5 (KLF5) is resistant to degradation by glycogen synthase kinase $3 \beta$ (GSK3 $\beta$ ) and the E3 ubiquitin ligase F-box and WD repeat domain-containing 7 $\alpha$ (FBW7 $\alpha$ ). J Biol Chem 2014;289:5997-6005.

31 Nayak L, Goduni L, Takami Y, Sharma N, Kapil P, Jain MK, Mahabeleshwar GH: Kruppel-like factor 2 is a transcriptional regulator of chronic and acute inflammation. Am J Pathol 2013;182:1696-1704.

-32 Sun J, Luan Y, Xiang D, Tan X, Chen H, Deng Q Zhang J, Chen M, Huang H, Wang W, Niu T, Li W, Peng H, Li S, Li L, Tang W, Li X, Wu D, Wang P: The 11S Proteasome Subunit PSME3 Is a Positive Feedforward Regulator of NF- $\kappa B$ and Important for Host Defense against Bacterial Pathogens. Cell Rep 2016;14:737-749. 